\title{
Mitochondrial role in the neonatal predisposition to developing nonalcoholic fatty liver disease
}

\author{
Peter R. Baker II' and Jacob E. Friedman ${ }^{2,3,4}$ \\ ${ }^{1}$ Section of Clinical Genetics and Metabolism, Department of Pediatrics, ${ }^{2}$ Section of Neonatology, Department of Pediatrics, ${ }^{3}$ Department of Biochemistry and Molecular Cenetics, and ${ }^{4}$ Department of \\ Medicine, Division of Endocrinology, Diabetes and Metabolism, University of Colorado Anschutz Medical Campus, Aurora, Colorado, USA.
}

\begin{abstract}
Nonalcoholic fatty liver disease (NAFLD) is a global epidemic in obese children and adults, and the onset might have fetal origins. A growing body of evidence supports the role of developmental programming, whereby the maternal environment affects fetal and infant development, altering the risk profile for disease later in life. Human and nonhuman primate studies of maternal obesity demonstrate that risk factors for pediatric obesity and NAFLD begin in utero. The pathologic mechanisms for NAFLD are multifactorial but have centered on altered mitochondrial function/dysfunction that might precede insulin resistance. Compared with the adult liver, the fetal liver has fewer mitochondria, low activity of the fatty acid metabolic enzyme carnitine palmitoyl-CoA transferase-1, and little or no gluconeogenesis. Exposure to excess maternal fuels during fetal life uniquely alters hepatic fatty acid oxidation, tricarboxylic acid cycle activity, de novo lipogenesis, and mitochondrial health. These events promote increased oxidative stress and excess triglyceride storage, and, together with altered immune function and epigenetic changes, they prime the fetal liver for NAFLD and might drive the risk for nonalcoholic steatohepatitis in the next generation.
\end{abstract}

\section{Introduction}

Nonalcoholic fatty liver disease (NAFLD) describes a spectrum of liver disease characterized by progressive steatosis and inflammation, leading to the more serious nonalcoholic steatohepatitis (NASH), which can precede cirrhosis and liver cancer (1). NAFLD is now the most common liver disease worldwide, affecting $80 \%$ of obese adults and $40 \%$ of all adults in the United States (2). There are limited insights into the origin and underlying mechanisms of pediatric-onset NAFLD, which is arguably more severe and aggressive (3). NAFLD prevalence ranges from $0.7 \%$ in young children aged 2-4 years, to approximately $40 \%$ in obese children (4). The prevalence of NAFLD increased approximately 3-fold from the late 1980 s to the present (4). Young children are now more likely than ever to experience early onset and increased severity of obesity and, importantly, in utero exposure to maternal obesity and insulin resistance, which exacerbate NAFLD risk (5).

The pathologic mechanisms underlying NAFLD are complex and multifactorial but center on altered mitochondrial function that might precede the development of NAFLD (6). Human studies describe increased intrahepatocellular lipid storage in infants born to obese mothers $(7,8)$. In later life, these offspring are at a higher risk of progressing to obesity, NAFLD, cardiovascular disease, and hepatic carcinoma (9-11). The in utero environment's critical importance to pediatric NAFLD is illustrated in a cross-sectional study of 538 children with biopsy-proven NAFLD.

Conflict of interest: JEF is a consultant to the scientific advisory board of Janssen Pharmaceuticals.

Reference information: J Clin Invest. 2018;128(9):xxxx-yyyy.

https://doi.org/10.1172/JCl120846
Those born with higher or lower birthweight had a 2-fold greater incidence of NAFLD, even after adjusting for childhood BMI (12). Here, we review the literature supporting the influence of gestational exposures, including maternal obesity, Western-style diet (WSD), and gestational diabetes mellitus (GDM), on mitochondrial function in the pathophysiology of pediatric NAFLD and progression to NASH. Through animal models, including nonhuman primates (NHPs), and limited human investigations, we demonstrate the importance of fetal metabolic programming in NAFLD, especially as it pertains to mitochondrial function. Finally, we review potential interventions preventing the early development of NAFLD in at-risk offspring.

\section{Neonatal predisposition to pediatric NAFLD}

Growing evidence suggests that individuals who develop pediatric NAFLD are born with predisposed risk. Severity of childhood NAFLD correlates with maternal obesity (13), birthweight (14), and shorter duration of breastfeeding $(13,15)$, even after adjusting for childhood BMI. The fetal programming hypothesis, that environmental conditions during critical periods influence longterm physical development, was first suggested by David Barker regarding maternal undernutrition and low-birthweight infants (16). From the early 1990 s to the present, however, human epidemiologic studies combined with functional studies in animal models have demonstrated that increased maternal BMI, insulin resistance, and high-fat diet (HFD) all contribute to increased risk of metabolic disease in offspring later in life. Mothers entering pregnancy with preexisting insulin resistance, such as those with obesity or GDM or consuming a WSD (high in both fat and sugar), display an overabundance of maternal metabolic sub- 


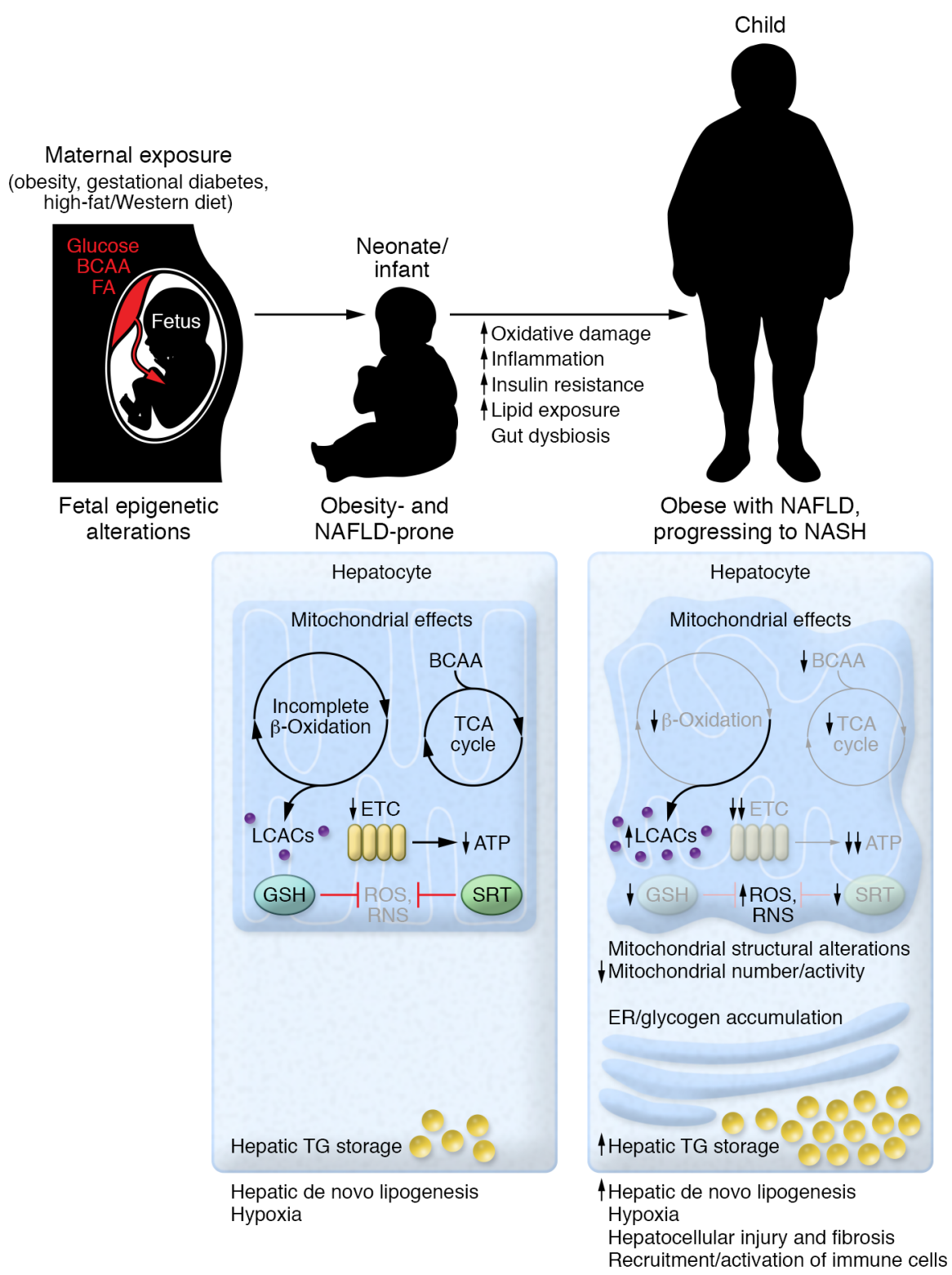

Figure 1. Overview of the early development of NAFLD and progression to NASH in offspring of overnourished mothers. Based on nonhuman primate and limited human data, offspring exposed to overnutrition in utero have increased hepatic lipid storage and de novo lipogenesis, coupled with incomplete $\beta$-oxidation and diminished electron transport chain (ETC) activity, leading to accumulation of long-chain acylcarnitines (LCACs) and diminished ATP production. Anaplerosis through branched-chain amino acid (BCAA) catabolism compensates for limitations in TCA cycle intermediates. This fuel overload and excess in lipid result in production of ROS. Damage is mitigated by glutathione (CSH) and upregulation of SIRT1 (SRT). Hepatic apoptosis is minimal (but existing). As time progresses and damage, inflammation, and lipid accumulation worsen, mitochondria develop structural abnormalities and diminish in content and activity. This is exacerbated by diet and the microbiome. $\beta$-Oxidative function and ETC activity worsen. Intramitochondrial lipid and acylcarnitine accumulation leads to increased ROS and reactive nitrogen species (RNS) production. Glycogen and smooth ER accumulate. BCAA catabolism is less efficient and is unable to supplement the TCA cycle, and GSH is no longer able to dampen oxidative damage. Inflammation due to infiltration and activation of immune cells and apoptosis worsen, leading to liver injury and fibrosis. FA, fatty acid; TG, triglyceride.

strates (17). The fetus might be especially vulnerable to steatosis because immature fetal adipose depots that buffer the excess transplacental lipids or other fuels in maternal obesity are not available until late in gestation. Excess substrates contribute not only to excess fetal growth but also to increased risk for childhood obesity and metabolic disease. Maternal metabolites that indicate altered mitochondrial function and are associated with maternal overnutrition include excess glucose, fatty acids (FAs), triglycerides, branched-chain amino acids (BCAAs), and longchain acylcarnitine species. These are elevated in maternal plasma during pregnancy (17), are found in cord blood at birth (18), and are intimately associated with maternal BMI, insulin resistance in pregnancy, high maternal dietary fat intake, and high infant birthweight (19).

Limited human data suggest that the maternal environment is important for NAFLD pathogenesis extremely early in life. The most direct evidence derives from a handful of studies in offspring of mothers with diabetes (gestational and pregesta- tional) and/or obesity. In one study, 105 neonates (mean age 11 days) of mothers with a BMI ranging from underweight to obese $\left(16-36 \mathrm{~kg} / \mathrm{m}^{2}\right)$ were characterized based on adipose tissue and hepatic lipid content using MRI and NMR spectroscopy (8). Neonatal hepatic fat content, as well as total adiposity, correlated with maternal BMI after correcting for infant sex and gestational age (8). Using a noninvasive MRI method, we described a $68 \%$ increase in hepatic lipid content in the 2-week-old offspring of mothers with obesity and GDM versus normal-weight mothers (7). In this study and the former study, follow-up hepatic MRIs were not performed at a later age. Therefore, neither the persistence of increased intrahepatic fat nor the risk of these infants going on to develop NASH was assessed. Finally, in another study, $78 \%$ of stillborn offspring of obese mothers with diabetes $(n=33)$ had evidence of hepatic steatosis versus $17 \%$ of stillborn offspring of nondiabetic mothers $(n=48)$ (20). Diabetes in this study was predominantly gestational $(n=22)$, but mothers with preexisting diabetes were included as well (20). 
Table 1. Genes associated with mitochondrial function and risk for NAFLD

\begin{tabular}{|c|c|}
\hline Gene & Function \\
\hline ADIPOO (160) & Stimulates fat metabolism via AMPK pathway activation and mitochondrial biogenesis stimulation (161) \\
\hline GCKR (162) & $\begin{array}{l}\text { Regulates hepatic glucokinase in response to fructose metabolites; glucokinase is important in mitochondrial apoptotic processes (163), } \\
\text { mitochondria-associated AMPK signaling, and oxidative stress (164) }\end{array}$ \\
\hline HO-1 (HMOX1) (170) & $\begin{array}{l}\text { Hypoxic response gene that directly regulates mitochondrial electron transport chain function via FOX01 (171) and stimulates mitochondrial } \\
\text { biogenesis (172); together with mitochondrial integrity, mediates lipid droplet formation in stress (173) and HFD via SIRT1 (174) }\end{array}$ \\
\hline MBOAT7 (177) & $\begin{array}{l}\text { Involved in mitochondrial phospholipid metabolism specifically contributing to regulation of arachidonic acid, affected in oxidative stress and } \\
\text { inflammation; resides in mitochondrial membrane (178) }\end{array}$ \\
\hline MNSOD (SOD2) (179-181) & $\begin{array}{l}\text { Binds to superoxide by-products of oxidative phosphorylation and converts them to hydrogen peroxide and diatomic oxygen; directly involved in } \\
\text { the oxidative stress response (182); enzyme activity directly linked to } \operatorname{NAFLD}(180,181)\end{array}$ \\
\hline PPARGC1A (PGC1A) (189) & Key upstream transcriptional regulator of mitochondrial metabolism and biogenesis; epigenetically regulated in the development of NAFLD (67) \\
\hline SAMM50 $(160,190)$ & $\begin{array}{l}\text { Mitochondrial membrane transporter that plays a crucial role in maintenance of the structure of mitochondrial cristae and proper assembly of } \\
\text { mitochondrial respiratory chain complexes }(191,192)\end{array}$ \\
\hline TMGSF2 $(162,193,194)$ & $\begin{array}{l}\text { Transcriptional regulator of liver fat metabolism influencing triglyceride secretion and hepatic lipid droplet content (195), impacting lipid used in } \\
\text { mitochondrial FA oxidation (196) }\end{array}$ \\
\hline UCP2 (197) & $\begin{array}{l}\text { Separates oxidative phosphorylation from ATP synthesis with energy dissipated as heat (mitochondrial proton leak); directly related to obesity } \\
\text { and insulin resistance and functionally linked to mitochondrial pathophysiology in NAFLD (198-200) }\end{array}$ \\
\hline
\end{tabular}

No neonatal studies looking at prospective development of NAFLD or testing biomarkers in the setting of increased neonatal hepatic lipid accumulation in humans have been reported. Early detection of NAFLD is challenging because of difficulties of tissue sampling and of performing potentially invasive studies on otherwise asymptomatic children. The Avon Longitudinal Study of Parents and Children (ALSPAC) demonstrated that offspring of insulin-resistant mothers were at higher risk for signs of NAFLD at 17 years of age versus offspring of mothers without insulin resistance (21). Increased maternal BMI was also associated with NAFLD in offspring, largely owing to its correlation with offspring adiposity (21). The Western Australian Pregnancy (Raine) Cohort Study reported similar results: increased maternal pre-pregnancy BMI conferred sex-specific risk for NAFLD in offspring at 17 years of age (22). Offspring of obese or diabetic mothers born with intrauterine growth restriction (IUGR) have a predisposition to develop NAFLD (23). Undernutrition in pregnancy can also result in IUGR in offspring and predispose offspring to develop metabolic syndrome and NAFLD (23).

Noninvasive analyses examining blood from offspring of obese and/or insulin-resistant mothers offer more insight into potential mitochondrial mechanisms of hepatic lipid accumulation in the setting of fetal programming. The Hyperglycemia and Adverse Pregnancy Outcome (HAPO) study, one of the largest studies to date, examined metabolites in neonates in relation to maternal obesity (18). Metabolomic analyses on cord blood from 1,600 mother-infant pairs spanning four ethnicities showed that mater- nal BMI was associated with cord blood BCAAs and their catabolites propionylcarnitine (C3), butyrylcarnitine/isobutyrylcarnitine (C4/Ci4), and isovalerylcarnitine (C5) (18). Maternal glucose was associated with cord blood levels of ketone 3-hydroxybutyrate, its carnitine ester, 3-hydroxy-decanoyl carnitine (C10OH or C8DC), and glycerol (18). These biomarkers are classically linked to hepatic and skeletal muscle mitochondrial dysmetabolism, as well as insulin resistance and risk of type 2 diabetes in adults.

In our unpublished analysis of neonatal plasma at 48 hours of life, we found markers of incomplete mitochondrial lipid oxidation (medium-chain and dicarboxylic acylcarnitines) and higher C5 relative to neonatal adiposity in offspring of overweight/obese, but not normal-weight, mothers (24). C5 is a marker of insulin resistance and related compensatory increased BCAA catabolism $(25,26)$. Interestingly, our proteomic analysis revealed enrichment of the BCAA catabolism pathway relative to neonatal adiposity in offspring of overweight/obese, but not normal-weight, mothers (24).

Stem cells from offspring of obese mothers might also provide clues regarding in utero programming of altered mitochondrial function. Using umbilical cord-derived mesenchymal stem cells (MSCs), we demonstrated relationships between maternal pre-pregnancy BMI, maternal circulating lipids during pregnancy, neonatal adiposity, and adiposity gain in the first few months of life with markers of altered mitochondrial function (27-30). It should be noted that although the MSCs studied were induced to differentiate toward adipocytes and myocytes, these same 

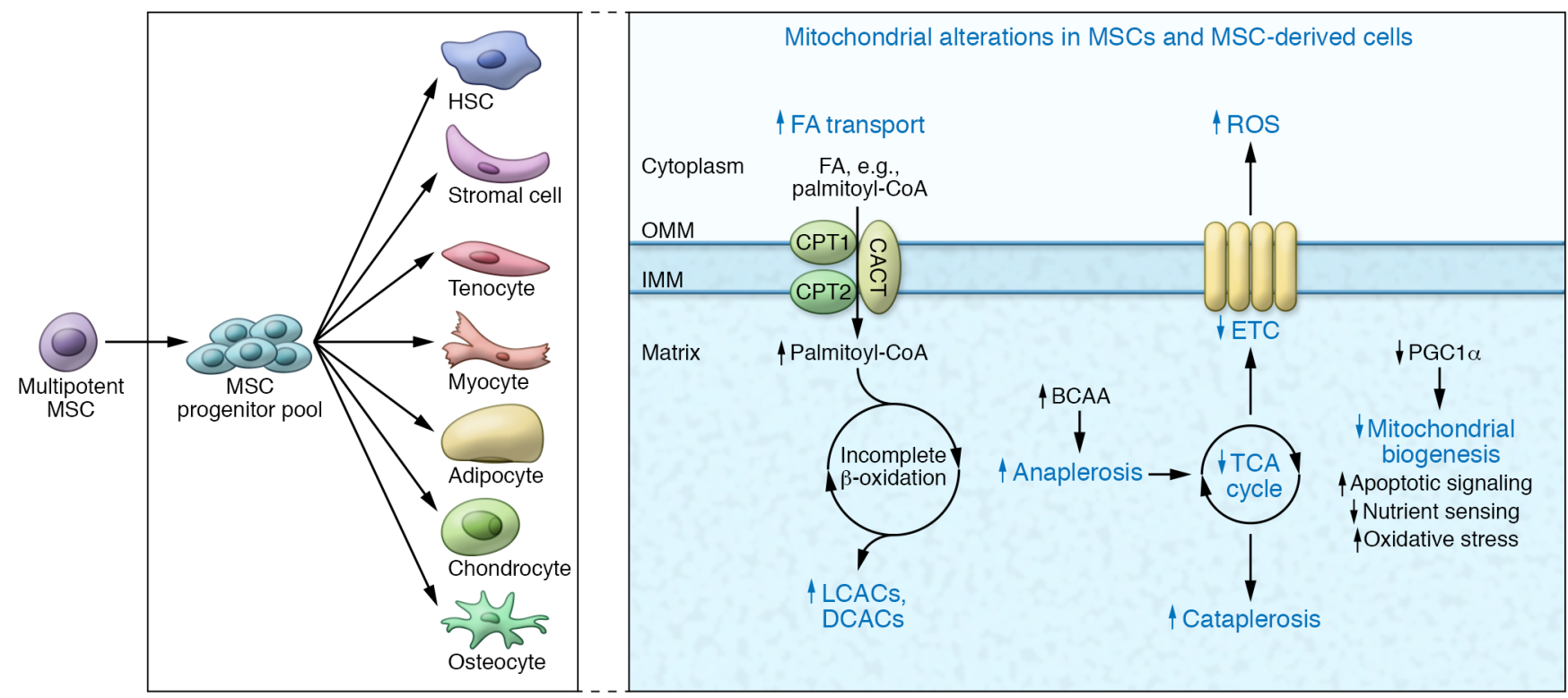

Figure 2. Evidence of Fetal Programming in Human Mesenchymal Stem Cells. Human mesenchymal stem cells (MSCs), progenitors to hepatic stellate cells (HSCs), demonstrate evidence of fetal programming in relation to maternal obesity, maternal circulating lipids, and neonatal adiposity and adiposity gain over time. These cells have exhibited lipid transport and accumulation, incomplete $\beta$-oxidation, increased anaplerosis, and diminished ETC activity. Oxidative stress is coupled with increased GSH metabolism, and gene expression indicates alterations in nutrient sensing along with increased apoptotic and inflammatory signaling. OMM, outer mitochondrial membrane; IMM, inner mitochondrial membrane; CACT, carnitine-acylcarnitine translocase; DCAC, dicarboxylic acylcarnitines.

precursor cells are known to populate the liver as hepatic stellate cells, which are intricately involved in hepatic fibrosis $(31,32)$. In myocyte-differentiated MSCs, accumulation of long-chain and dicarboxylic acylcarnitines correlated with neonatal adiposity particularly in offspring of obese mothers (27). In differential gene expression analysis, we observed that pathway enrichment for multiple metabolic processes corresponds to maternal BMI (27). Adipocyte-differentiated MSCs demonstrated broad changes in mitochondrial gene expression related to maternal FA levels in the second trimester. There was upregulation of multiple electron transport chain (ETC) genes coupled with downregulation of genes related to mitochondrial biogenesis, including CREBBP, EP300, and PPARA (27). Also, pathway enrichment was observed for nutrient-sensing pathways, including PI3K/AKT and AMPK, relative to maternal BMI (27).

Rapid gain in adiposity in the first 5 months of life is a known risk factor for childhood obesity and metabolic syndrome and is a risk marker for NAFLD $(33,34)$. In infants with rapid gains in adiposity, we found markers of incomplete lipid oxidation and upregulation of membrane lipid transport genes in their MSC-derived adipocytes (28). We further found alterations in analytes and genes in the glutathione cycle, including higher cysteine concentrations and upregulation of GCLC, the rate-limiting step in glutathione synthesis and ophthalmate generation (28). Oxidative stress was greater, as suggested by upregulation of SOD2 and HIF1 gene expression in MSCs (28). Taken together, these findings suggest that rapid gain in adiposity in an infant's first months of life corresponds with potentially lipotoxic alterations in their stem cells. More germane to the discussion of hepatic disease, we also found upregulation of genes in the Kyoto Encyclopedia of Genes and Genomes (KEGG) NAFLD disease pathway as well as upregulation of mitochondrial oxidative phosphorylation genes relative to rapid infant gain in adiposity (28).

\section{Hepatocellular changes and fetal programming in NAFLD}

Fetal hepatic metabolism is unique in that the fetus develops in a low-oxygen environment with limited capacity for lipid and amino acid oxidation until birth $(35,36)$. Compared with adult livers, the fetal liver has fewer mitochondria, lower activity of carnitine palmitoyl-CoA transferase-1 (CPT1, the enzyme responsible for mitochondrial FA transport), and little or no gluconeogenesis (37). These data suggest that the fetal liver is uniquely vulnerable to dysregulated fuel metabolism caused by excess fuel exposure due to maternal obesity or poor diet. However, few studies have investigated whether impaired mitochondrial function exists in the fetus, despite numerous studies in postnatal human and animal models (38-40).

We have described lipotoxic effects of maternal HFD in fetal NHP livers, including increased hepatic triglyceride storage and oxidative stress early in the third trimester, consistent with the development of NAFLD (41). PPARGC1A gene expression and PGC1 $\alpha$ enzyme activity were increased (41), suggesting that mitochondrial biogenesis upregulates possibly in response to increased oxidative stress. Insulin-resistant NHP dams on HFD demonstrated increased body mass, higher triglycerides, and increased placental cytokines (42). Offspring of these dams had increased liver triglycerides and upregulated hepatic de novo lipid synthesis at 1 year of life despite weaning to a healthy diet (42). Irreversible hepatic inflammation was observed even in the absence of obesity 
or insulin resistance (42). NHP dams on HFD also had higher plasma omega-6/omega-3 FA ratios (43), reflecting higher arachidonic acid (omega-6) versus docosahexaenoic acid (omega-3) and eicosapentaenoic acid (omega-3). Not only is this detrimental to fetal brain development, higher concentrations of omega-6 FAs (specifically arachidonic acid) have been associated with NAFLD-related mitochondrial dysfunction involving downregulation of PPARA, a key transcriptional regulator of FA oxidation, as well as downstream target genes $C P T 1 A$ and $A O X$, which are intricately involved in mitochondrial and peroxisomal FA oxidation (44). The fetal offspring of these HFD-fed NHP dams had a higher omega-6/ omega-3 ratio versus controls, which significantly correlated to maternal plasma omega-6/omega-3 ratio and maternal hyperinsulinemia (43). These fetuses had a higher amount of hepatic apoptosis (43). Hepatic apoptosis has been described in NASH (45) and is influenced by increased FA exposure that sensitizes hepatocytes to cytotoxicity mediated by TNF-related apoptosis-inducing ligand (TRAIL) (46). Changing the mother to a control diet in the subsequent pregnancy mitigated hepatic apoptosis in the fetus (43).

In mice, a similar relationship exists between maternal obesity and HFD and offspring risk for altered mitochondrial function and NAFLD. Adult offspring of HFD-fed mouse dams had NAFLD (47) and upregulated expression of SREBP1 (48). This was true for dams fed an HFD during gestation and/or lactation and was exacerbated when offspring were weaned to HFD themselves (48). Hepatic mitochondrial dysfunction was reported in offspring of HFD-fed mouse dams, with close ties to oxidative damage, lipid storage, altered methylation patterns, and altered mitochondria-related gene expression (including Ppara, Ppargc1b, and Fgf21) in offspring adolescence $(49,50)$ and adulthood $(51,52)$. As in the NHP mod$\mathrm{el}$, the offspring immune system in mice is altered, contributing to the programmed pathogenesis in NAFLD (53).

In rats, evidence suggests that maternal HFD as well as fetal hypoxia programs offspring predisposition for NAFLD. During both pregnancy and lactation, maternal HFD resulted in reduced hepatic mitochondrial DNA (mtDNA) content and activity of PGC1 $\alpha$, with pronounced insulin resistance, hyperleptinemia, and hepatic fat accumulation in adult offspring maintained on HFD (54). Muscle glycolytic metabolism increased, FA oxidation with palmitoylcarnitine (C16) decreased, and hepatic triglyceride content increased in HFD-fed offspring of HFD-fed dams; these effects increased with age (55). Similar changes in hepatic fat content and oxidative stress occurred in offspring exposed to maternal hypoxia with IUGR (56), particularly in HFD-fed rat offspring (57). Mechanistic links have been made with nutrient sensing, inflammatory response to oxidative stress, and mitochondria-related gene expression (58-60).

\section{Epigenetic modifications in fetal programming of NAFLD}

Epigenetic studies in mice show that exposure to maternal overnutrition is associated with hepatic DNA methylation (61) and histone posttranslational modifications (62) that are first evident at 5 weeks of life and persist after weaning to a low-fat diet even into adulthood. Many gene targets relate to mitochondrial metabolism and metabolic regulation. In humans, studies of epigenetic changes in NAFLD are largely confined to adults but can still provide some insight into epigenetic modifications present in pediatric NAFLD. One of the few pediatric studies investigating epigenetic changes in NAFLD-affected children found that elevations of alanine aminotransferase were associated with HIF3A methylation, suggesting that epigenetic changes in the oxidative stress response predispose children to NAFLD (63). In adult humans, evidence is more robust that epigenetic alterations can affect mitochondrial metabolism specifically through differential methylation of genes related to mitochondrial and lipid metabolism (64). Mitochondrial mRNA and protein expression, particularly of NADH dehydrogenase 6 (MT-ND6), was significantly decreased in livers of NASH patients versus simple steatosis patients (65). In genomic DNA, the epigenetic marker 5-hydroxymethylcytosine was related to liver mtDNA copy number and PPARGC1A mRNA levels in NAFLD patients versus control groups (66). In biopsied livers from NAFLD patients, PPARGC1A methylation (and diminished gene expression) correlated positively with insulin resistance, and methylation of TFAM (transcription factor A, mitochondrial) has correlated negatively with insulin levels (67). Mitochondrial content is diminished in NAFLD livers, inversely correlating with HOMA-IR (a measure of insulin resistance), fasting glucose, insulin, and PPARGC1A promoter methylation (67).

Acetylation patterns also play a role in programmed NAFLD pathogenesis. In our NHP model, maternal HFD induced fetal NAFLD and modulated fetal hepatic SIRT1 histone and protein deacetylase activity (68). SIRT1 is both a lysine deacetylase and a crucial sensor of cellular metabolism. We have demonstrated that in utero exposure to a maternal HFD, but not necessarily maternal obesity, increased fetal H3K14 histone acetylation with a concomitant decrease in SIRT1 expression and diminished in vitro protein and histone deacetylase activity (68). This was tied to altered expression of known downstream effectors that are deregulated in NAFLD and modulated by SIRT1, including PPARA, PPARG, SREBF1, CYP7A1, $F A S N$, and $S C D$ (68). Further studies found that inhibiting SIRT1 signaling in human fetal hepatocytes rapidly led to increases in intracellular glucose and lipid levels (69). Both de novo lipogenesis- and gluconeogenesis-related genes were upregulated upon SIRT1 inhibition (69). The AKT/FOXO1 pathway, a negative regulator of gluconeogenesis, decreased in the human fetal hepatocytes in which SIRT1 was inhibited, and gluconeogenesis increased (69).

Taken together, evidence from NHP, mouse, and rat models points to a maternal HFD programming offspring mitochondrial function, increasing offspring ROS and inflammation, and promoting offspring hepatic lipid storage from the fetus into adulthood. These effects are exacerbated by maternal HFD during lactation and the offspring's consumption of HFD after weaning and mediated, at least in part, by genetic and epigenetic factors, with epigenetic modifications largely dependent on maternal dietary exposures (Figure 1).

\section{Mitochondrial health and lipid metabolism in established NAFLD/NASH}

While the development of NAFLD is no doubt multifactorial, mitochondrial structure and function have a central role in disease pathogenesis. While one of the most cited genes associated with NAFLD is PLAPN3, a triglyceride processing gene (70), in multiple human studies (Table 1), static alterations of mitochondria-associated genes lay a foundation for mitochondrial health and are 
thought to contribute strongly to NAFLD/NASH susceptibility. Genetic polymorphisms associated with NAFLD (Table 1, column 1) are found largely in genes with a connection to mitochondrial function (Table 1, column 2). With this genetic foundation comes maternal in utero exposure and hepatic mitochondrial exposures postnatally, causing mitochondrial structure and function to devolve in offspring as the disease progresses (Figure 1). In one of the few published studies of its kind, an in-depth description of mitochondrial ultrastructure was conducted in liver biopsy specimens from ten children aged 2-14 years with previously clinicopathologically diagnosed NASH (71). Besides steatosis and typical inflammatory, apoptotic, and fibrotic changes seen in NASH, investigators found megamitochondria, loss of mitochondrial cristae, and the presence of linear crystalline inclusions within the mitochondrial matrix causing mitochondrial deformations (71), suggestive of hepatic lipotoxicity. These inclusions have been reported in other inflammatory hepatic disorders $(13,14,72,73)$, and are correlated with oxidative stress $(15,74,75)$. Foamy cytoplasm of hepatocytes was found (71), representing proliferation of smooth ER and glycogen accumulation. The perivascular space of Disse was dilated and contained activated hepatic stellate cells as well as collagen fiber bundles (71). Fat droplets have also been reported within the mitochondrial matrix (76), suggesting intact FA transport uncoupled from fat utilization for energy. These findings are similar in adults with NAFLD/NASH, suggesting potentially similar pathophysiology.

Obesity is associated with increased peripheral insulin resistance and increased adipocyte lipolysis, which overload the hepatocyte mitochondria (77). During NAFLD, FA oxidation increases the amount of acetyl-CoA into the tricarboxylic acid (TCA) cycle, but when mitochondrial ETC capacity is exceeded despite an increase in mitochondrial biogenesis, inefficient respiration and increased oxidative stress occur that further damage mitochondria and oxidative capacity. Lipotoxicity, characterized by production of ROS, and related inflammation associated with mitochondrial dysfunction are well reported in NAFLD and NASH (78-80). Incomplete mitochondrial and peroxisomal lipid oxidation and/or ETC dysfunction lead to lipid peroxidation and oxidative damage (81-83). These changes might even precede lipid storage and insulin resistance. In the obese, hyperphagic Otsuka Long-Evans Tokushima Fatty (OLETF) rat model, researchers found significant hepatic mitochondrial dysfunction as measured by reduced hepatic CPT1 activity, FA oxidation, and cytochrome $c$ protein content versus controls at 5 weeks of life (6). This finding occurred in the setting of normal hepatic triglycerides and normal serum insulin and glucose. It was not until 8 weeks of age that hepatic triglycerides began to significantly accumulate, followed by the development of insulin resistance at 13 weeks of age. NAFLD progressively worsened over the life of the OLETF animals. They developed hepatocyte ballooning, fibrosis, elevated transaminases, hepatic mitochondrial ultrastructural abnormalities, and increased hepatic oxidative stress following the initial disease development between 5 and 13 weeks of age. Measures of hepatic mitochondrial content and function, including hydroxyacyl-CoA dehydrogenase enzyme activity, citrate synthase activity, and mitochondrial CPT1 content, progressively declined and were significantly reduced by 40 weeks of age (6).
Throughout NAFLD progression, mitochondria-specific processes including FA oxidation, ketogenesis, ETC component activity, oxygen consumption, and mtDNA content change (81). FA oxidation and ketogenesis are increased in every stage of NAFLD progression $(81,84,85)$, possibly as a result of stimulation of upstream signaling of leptin, PPAR $\alpha$, and PGC1 $\alpha$ (86-89), worsening of hepatic insulin resistance (74), and/or upregulation of genes in the mitochondrial FA oxidation pathway (e.g., CPT1) $(90,91)$. Complex I and IV activity is typically unchanged or reduced and mtDNA content and damage increase as NAFLD worsens (81). Hepatic ATP levels are diminished (92) and oxidative capacity is limited, particularly in the setting of HFD (93).

\section{Mitochondrial intermediary metabolism in NAFLD/NASH}

Mitochondrial pathways beyond FA metabolism and the ETC play a role in the pathogenesis of NAFLD (Figure 2). In NAFLD, there is an increase in anaplerosis and cataplerosis, processes that replenish and utilize intermediates of the TCA cycle, respectively (82, $94,95)$. Anaplerotic processes allow the hepatocyte to maintain coupling of glycolysis and/or FA oxidation with the mitochondrial ETC using molecules like amino acids to enter the TCA cycle and replenish its intermediates. Cataplerotic processes include gluconeogenesis, which utilizes TCA intermediates to make glucose. In adult NAFLD, a 50\% higher rate of lipolysis and a 30\% higher rate of gluconeogenesis are observed, with positive correlations between hepatic lipid content and both mitochondrial oxidative and anaplerotic fluxes (94). This is enhanced with hepatic insulin resistance (95). Mitochondrial oxidative metabolism is approximately 2-fold greater in those with NAFLD, providing a potential link between hepatic lipid content, oxidative stress, liver damage, and increased rates of anaplerosis and cataplerosis (94). This is mediated in part by phosphoenolpyruvate carboxykinase (PEPCK) (82), and is likely a compensatory mechanism to maximize mitochondrial respiratory capacity (96). Notably, genes and substrates for the gluconeogenic pathway are upregulated in the liver of fetuses from obese NHP dams (41).

BCAA metabolism is modulated for anaplerosis in NAFLD. Ketoisocaproate is a catabolite of leucine, a BCAA metabolized in mitochondria, and a key biomarker of insulin resistance and metabolic syndrome (25). Ketoisocaproate levels have been reported to be inversely related to the progression of NAFLD (97) and inversely related to alanine aminotransferase and $\gamma$-glutamyltransferase levels in obese adults (98). Ketoisocaproate decarboxylation is decreased in steatohepatitis $(97,99)$. The end products of BCAA catabolism directly supplement the TCA cycle in anaplerosis, a process necessary in the setting of incomplete $\beta$-oxidation in metabolic syndrome $(26,100)$. BCAAs have been positively associated with NAFLD and its associated progression in adults and adolescents (101-103), with downregulation of gene expression in the BCAA catabolism pathway $(101,102)$. If anaplerosis is a compensatory mechanism in NAFLD (104), progression to NASH might occur in part when this mechanism fails to supply the TCA cycle with carbon substrate.

One-carbon metabolism is closely related to hepatic lipid accumulation. It is known to affect the development of NASH through the modulation of $S$-adenosylhomocysteine hydrolase; 
increased concentration is associated with epigenetic modification and transcriptional dysregulation $(105,106)$. One classic animal model of NASH, methionine and/or choline restriction (MCR), illustrates the importance of the one-carbon pathway in maintaining healthy levels of hepatic lipid content (107). MCR rats demonstrated altered mitochondrial function in ETC complexes I and V, oxidative stress with increased ROS production, and increased hepatic fat content in the liver (108-110) without obesity. Diminished endogenous hepatic phosphatidylcholine in MCR plays a role in NAFLD pathogenesis, through both altered mitochondrial membrane fluidity (111) and inefficient formation of lipoproteins for FA export (112). Choline deficiency is related to depletion of cardiolipin $(108,109)$, which has been implicated in multiple aspects of mitochondrial dysfunction in NAFLD, including oxidative stress, bioenergetics, and mitochondrial-induced apoptosis (113). The one-carbon pathway is closely linked to glutathione metabolism (114), thereby implicating the major role of altered antioxidant activity in the progression of NAFLD to NASH. Exactly what role each pathway in one-carbon metabolism plays in the assembly and retention of hepatic triglycerides is not clear.

\section{Mitochondrial oxidative stress and apoptosis in NAFLD/NASH}

Enhanced FA oxidation without concomitant upregulation of ETC activity induces ROS overproduction upstream of cytochrome $c$ oxidase (115). Glutathione and superoxide dismutase mitigate mitochondrial oxidative stress but are reduced as NAFLD progresses to NASH (115). Notably, in the fetus, endogenous antioxidant enzymes and placental transfer of antioxidant micronutrients or enzymes are limited until late in gestation in preparation for the oxidative stress associated with labor and birth (116). Oxidative stress promotes inflammation by releasing cytokines like CXCR3, which actively inhibit mitochondrial function and dynamics (117). Influx of lipids also increases stress from reactive nitrogen species (RNS) generated by TNF- $\alpha$-stimulated CYP2E1 and inducible NO synthase $(118,119)$, which is central to the progression of NAFLD to NASH (120). Increases in RNS correspond to worsening hypoxic conditions and nitrosylation of mitochondrial proteins, resulting in uncoupled respiration along with depressed active respiration, complex I and cytochrome $c$ oxidase activity, and mitochondrial membrane potential $(118,119)$. Notably, placentas from obese, HFD-fed NHP dams showed evidence for hypoxia and increased cytokine production in the fetal circulation $(41,121)$.

The fire of this lipid oxidative stress has gasoline thrown onto it when individuals with NAFLD consume lipid and cholesterol. Ingestion of lipid in the setting of NAFLD worsens mitochondrial function, downregulates mitochondrial biogenesis, and promotes hepatocellular apoptosis (122). Exposure to saturated lipids like palmitate promotes apoptosis through the mitochondrial $\mathrm{B}$ cell lymphoma-2 (BCL-2) pathway (a regulator of mitochondrial permeability), prevents the release of cytochrome $c$ into the cytosol, binds to apoptotic peptidase activating factor-1, and controls NLRP1 inflammasome activation (123-126). Saturated FAs and ceramides contribute to cellular damage in NAFLD, while monounsaturated species and triglycerides might have a more protective role (127-129). Ceramides are the most directly damaging lip- id molecules (130), with contributions to mitochondrial-mediated oxidative damage and inhibition of oxidative phosphorylation specific to complexes II and IV $(131,132)$.

\section{Further clues to mechanisms in pediatric NAFLD/NASH}

The majority of information regarding the origins of NAFLD in children is based on animal models. Histologically, pediatric NASH is often associated with a unique pattern of inflammation in the periportal region, suggesting a strong inflammatory component (133-135). While the pathophysiologies of adult and pediatric NAFLD are not identical, evidence suggests there is an overlap (26). A recent study used metabolomics in infant cord blood to identify analytes associated with neonatal anthropometry, with the goal of finding biomarkers and candidate pathways in infant predisposition to obesity (136). Metabolites in energy production (TCA cycle intermediates) were associated with larger size at birth and higher levels of leptin and insulin-like growth factor-1, and the BCAA metabolite pattern was associated with larger birth size (136).

To date, no neonatal studies looking at prospective development of NAFLD or testing biomarkers in the setting of increased neonatal hepatic lipid accumulation have been reported. An analysis of cord blood samples found little correlation between blood markers (including lipids, transaminases, and hormones like leptin and adiponectin) and the ability to predict NAFLD later in adolescence (137). Obese adolescents with NAFLD have increased levels of circulating BCAAs $(103,138)$. These biomarkers are increased in the setting of insulin resistance (25) and indicate altered mitochondrial function in energy production in liver and skeletal muscle (26). Homocysteine and cysteine, one-carbon amino acids, are also elevated in the blood of children with NAFLD $(138,139)$. These biomarkers are linked to glutathione metabolism, and in NAFLD they are markers of oxidative stress typically mitigated by the mitochondria $(138,139)$. Small-molecule intermediary metabolites, including BCAAs and methionine, were elevated in serum of obese versus nonobese adolescents, as were FA oxidation intermediates and acylcarnitines, indicating incomplete $\beta$-oxidation $(140,141)$. Finally, fructose intake and metabolism also mediate development of pediatric NAFLD, with direct mitochondrial mechanisms. Hepatic dysfunction and hepatic lipid accumulation can occur when the liver is overloaded with fructose $(142,143)$. In pediatric NAFLD, fructose causes disruption of mitochondrial function, inflammation, and oxidative stress through increased fructokinase-mediated generation of fructose-1-phosphate and decreased concentrations of cellular ATP (143-145). Recent evidence suggests that fructose ingestion affects mitochondria at a transcriptional level, via hypomethylation of mtDNA (146).

Taken together, peripheral and hepatocyte-specific insulin resistance, hepatic lipid accumulation, and inflammation are tied intimately with altered mitochondrial function in postnatal development of NAFLD and its progression to NASH. These links occur in the context of exposures that impact mitochondrial function related to the maternal in utero environment, and/or transcriptional pathways altered by epigenetic changes in mitochondria-related genes, in addition to known genetic polymorphisms in pathways related to NAFLD risk. 


\section{Interventions to prevent altered mitochondrial function in NAFLD/NASH}

Current guidelines enforce lifestyle modification (diet and exercise) as the primary treatment for patients with established pediatric NAFLD (4), with clinical trials under way focused on modulation of metabolic pathways, inflammatory cascades, and/or mechanisms impacting fibrosis (147). Going beyond correlations to address causation and mechanisms of human disease, particularly in infancy, has been difficult because of tremendous variation in environment, nutrition, and lifestyle factors. The fetal overnutrition hypothesis suggests that maternal fuels contributing to childhood NAFLD and obesity are in greater abundance in maternal obesity and maternal WSD consumption (148). In the setting of maternal obesity complicated by GDM, a lower-carbohydrate (and by default higher-fat) diet is often recommended. Offspring of mothers with GDM are at risk for NAFLD in the neonatal period and possibly beyond (7). New GDM interventions including dietary therapy with higher complex carbohydrates, but lower simple carbohydrates and fat, have been shown to improve maternal lipid profiles and maternal adipocyte insulin sensitivity and to improve offspring adiposity (149). Studies are under way to see whether there is a beneficial effect on neonatal hepatic lipid storage. In children with NAFLD, breastfeeding during the infant period (150) and less refined carbohydrate intake (151) were beneficial, suggesting possible programming effects in infancy that last throughout life.

A typical WSD includes excessive amounts of simple sugars, saturated fats, and cholesterol, which are particularly proinflammatory. In humans, treatment with antiinflammatory omega-3 polyunsaturated FAs mitigated oxidative stress and altered mitochondrial function in NAFLD (152). Likewise, our recent studies in mice demonstrated that a potent dietary antioxidant, pyrroloquinoline quinone, which is found in high concentration in human breast milk (153), prevented early microbial dysbiosis and improved hepatic macrophage mitochondrial oxidative metabolism and NAFLD in adult offspring when administered to obese dams only during gestation and lactation (47). Fetal programming of NAFLD has also been prevented through maternal exercise. In HFD-fed rats, offspring from dams that had exercised had reduced body fat and were protected against HFD-induced hepatic steatosis (154). This was associated with upregulated markers of hepatic mitochondrial biogenesis (PGC1 $\alpha$ and TFAM) (154) and stimulation of FA oxidation and mitochondrial biogenesis (155). Metformin has been used to treat pediatric NAFLD safely and effectively, both alone and in combination with vitamin $\mathrm{E}$ therapy (156-158). Metformin is becoming increasingly used in GDM (159), and it has direct effects on adult mitochondrial metabolism associated with NAFLD (82), but its long-term effects in the offspring remain unstudied. However, because metformin crosses the placenta and affects a number of mitochondrial and nutrient-sensing mechanisms like AMPK, the mitochondrial ETC, and one-carbon metabolism, there could be untoward effects on offspring mitochondrial metabolism (159). Long-term follow-up studies are still needed.

\section{Conclusions}

In summary, the pathways to developmental programming of NAFLD suggest that the fetal liver, and hepatic mitochondrial function in particular, is uniquely susceptible to dysregulated fuel metabolism in the womb based on exposure to maternal obesity, diabetes, or WSD. In a fetal environment of low oxygen, low expression of mitochondria FA oxidation enzymes, and relatively low antioxidant activity, the fetal liver is particularly vulnerable to injury and poised for development of NAFLD. Increased hepatic lipid stores, disrupted mitochondria, and elevated oxidative stress, along with increased de novo lipogenic and gluconeogenic gene expression and epigenetic modifications in the liver, suggest that these factors contribute to lifetime risk of NAFLD and likely contribute to the severity and early onset of NAFLD in children. Although many factors contribute to disease progression, altering mitochondrial health through diet, exercise, and, potentially, targeted molecular therapies can help slow the rapid rise in pediatric NAFLD prevalence.

\section{Acknowledgments}

This research was supported by the University of Colorado Department of Pediatrics Child Health Research Career Development Award K12-HD068372 (P.R.B.), Colorado Nutrition and Obesity Research Center (NORC) grant P30DK048520 (P.R.B., J.E.F.), and Colorado Clinical and Translational Science Institute (CCTSI) pilot grant (P.R.B.). Non-human primate research was supported by the NIH-R24DK090964 (J.E.F).

Address correspondence to: Peter R. Baker II, University of Colorado Anschutz Medical Campus, 13123 E. 16th Avenue, Box 300, Aurora, Colorado 80045, USA. Phone: 303.724.8158; Email: peter. bakerii@ucdenver.edu.
1. Kopec KL, Burns D. Nonalcoholic fatty liver disease: a review of the spectrum of disease, diagnosis, and therapy. Nutr Clin Pract. 2011;26(5):565-576.

2. Cholankeril G, Perumpail RB, Pham EA, Ahmed A, Harrison SA. Nonalcoholic fatty liver disease: epidemiology, natural history, and diagnostic challenges. Hepatology. 2016;64(3):954.

3. Holterman AX, et al. Nonalcoholic fatty liver disease in severely obese adolescent and adult patients. Obesity (Silver Spring). 2013;21(3):591-597.

4. Vos MB, et al. NASPGHAN clinical practice guideline for the diagnosis and treatment of nonalcoholic fatty liver disease in children: recommendations from the expert committee on NAFLD (ECON) and the North American Society of Pediatric Gastroenterology, Hepatology and Nutrition (NASPGHAN). J Pediatr Gastroenterol Nutr. 2017;64(2):319-334.

5. Kelly AS, et al. Severe obesity in children and adolescents: identification, associated health risks, and treatment approaches: a scientific statement from the American Heart Association. Circulation. 2013;128(15):1689-1712.

6. Rector RS, et al. Mitochondrial dysfunction precedes insulin resistance and hepatic steatosis and contributes to the natural history of non-alcoholic fatty liver disease in an obese rodent model. J Hepatol. 2010;52(5):727-736.

7. Brumbaugh DE, et al. Intrahepatic fat is increased in the neonatal offspring of obese women with gesta- tional diabetes. JPediatr. 2013;162(5):930-936.e1.

8. Modi N, et al. The influence of maternal body mass index on infant adiposity and hepatic lipid content. Pediatr Res. 2011;70(3):287-291.

9. Houghton LC, et al. Maternal weight gain in excess of pregnancy guidelines is related to daughters being overweight 40 years later. $\mathrm{Am} \mathrm{J}$ Obstet Gynecol. 2016;215(2):246.e1-246.e8.

10. Hagström H, Stål P, Hultcrantz R, Hemmingsson $\mathrm{T}$, Andreasson A. Overweight in late adolescence predicts development of severe liver disease later in life: a 39 years follow-up study. J Hepatol. 2016;65(2):363-368.

11. Rinella M, Charlton M. The globalization of nonalcoholic fatty liver disease: Prevalence 
and impact on world health. Hepatology. 2016;64(1):19-22.

12. Newton KP, et al. Low and high birth weights are risk factors for nonalcoholic fatty liver disease in children. J Pediatr. 2017;187:141-146.e1.

13. Chandler RJ, et al. Mitochondrial dysfunction in mut methylmalonic acidemia. FASEB J. 2009;23(4):1252-1261.

14. Prieto I, et al. Tumor necrosis factor- $\alpha$, interleukin-1beta and nitric oxide: induction of liver megamitochondria in prehepatic portal hypertensive rats. World J Surg. 2005;29(7):903-908.

15. Ahishali E, et al. Electron microscopic findings in non-alcoholic fatty liver disease: is there a difference between hepatosteatosis and steatohepatitis? J Gastroenterol Hepatol. 2010;25(3):619-626.

16. Goyal NP, Schwimmer JB. The genetics of pediatric nonalcoholic fatty liver disease. Clin Liver Dis. 2018;22(1):59-71.

17. Jacob $S$, et al. Targeted metabolomics demonstrates distinct and overlapping maternal metabolites associated with BMI, glucose, and insulin sensitivity during pregnancy across four ancestry groups. Diabetes Care. 2017;40(7):911-919.

18. Lowe WL Jr., et al. Maternal BMI and glycemia impact the fetal metabolome. Diabetes Care. 2017;40(7):902-910.

19. Scholtens DM, et al. Metabolic networks and metabolites underlie associations between maternal glucose during pregnancy and newborn size at birth. Diabetes. 2016;65(7):2039-2050.

20. Patel KR, White FV, Deutsch GH. Hepatic steatosis is prevalent in stillborns delivered to women with diabetes mellitus. J Pediatr Gastroenterol Nutr. 2015;60(2):152-158.

21. Patel S, Lawlor DA, Callaway M, Macdonald-Wallis C, Sattar N, Fraser A. Association of maternal diabetes/glycosuria and pre-pregnancy body mass index with offspring indicators of non-alcoholic fatty liver disease. BMC Pediatr. 2016;16:47.

22. Ayonrinde OT, et al. Sex differences between parental pregnancy characteristics and nonalcoholic fatty liver disease in adolescents. Hepatology. 2018;67(1):108-122.

23. Alisi A, Panera N, Agostoni C, Nobili V. Intrauterine growth retardation and nonalcoholic fatty liver disease in children. Int JEndocrinol. 2011;2011:269853.

24. Baker PR II, et al. Metabolomic and proteomic analysis of neonatal plasma reveals novel evidence of early metabolic dysregulation from maternal obesity. Diabetes. 2016;65:A8.

25. Newgard CB, et al. A branched-chain amino acid-related metabolic signature that differentiates obese and lean humans and contributes to insulin resistance. Cell Metab. 2009;9(4):311-326.

26. Newgard CB. Metabolomics and metabolic diseases: where do we stand? Cell Metab. 2017;25(1):43-56.

27. Baker PR II, et al. Maternal obesity and increased neonatal adiposity are associated with altered infant mesenchymal stem cell metabolism. JCI Insight. 2017;2(21):e94200.

28. Baker PR, et al. Altered gene expression and metabolism in fetal umbilical cord mesenchymal stem cells correspond with differences in 5-monthold infant adiposity gain. Sci Rep. 2017;7(1):18095.

29. Boyle KE, Patinkin ZW, Shapiro AL, Baker
PR, Dabelea D, Friedman JE. Mesenchymal stem cells from infants born to obese mothers exhibit greater potential for adipogenesis: the Healthy Start BabyBUMP Project. Diabetes. 2016;65(3):647-659.

30. Boyle KE, et al. Maternal obesity alters fatty acid oxidation, AMPK activity, and associated DNA methylation in mesenchymal stem cells from human infants. Mol Metab. 2017;6(11):1503-1516.

31. Kordes C, Sawitza I, Götze S, Herebian D, Häussinger D. Hepatic stellate cells contribute to progenitor cells and liver regeneration. JClin Invest. 2014;124(12):5503-5515.

32. Tsai PC, et al. The therapeutic potential of human umbilical mesenchymal stem cells from Wharton's jelly in the treatment of rat liver fibrosis. Liver Transpl. 2009;15(5):484-495.

33. Breij LM, Kerkhof GF, Hokken-Koelega AC. Accelerated infant weight gain and risk for nonalcoholic fatty liver disease in early adulthood. $J$ Clin Endocrinol Metab. 2014;99(4):1189-1195.

34. Anderson EL, et al. Weight trajectories through infancy and childhood and risk of non-alcoholic fatty liver disease in adolescence: the ALSPAC study. J Hepatol. 2014;61(3):626-632.

35. Battaglia FC, Meschia G. Principal substrates of fetal metabolism. Physiol Rev. 1978;58(2):499-527.

36. Battaglia FC, Wilkening R, Meschia G. Unique organ specific characteristics of amino acid metabolism in early development. Trans Am Clin Climatol Assoc. 1995;106:141-149; discussion 149.

37. Kim SR, et al. Comparative metabolome analysis of cultured fetal and adult hepatocytes in humans. J Toxicol Sci. 2014;39(5):717-723.

38. Jelenik T, et al. Mechanisms of insulin resistance in primary and secondary nonalcoholic fatty liver. Diabetes. 2017;66(8):2241-2253.

39. Monsénégo J, et al. Enhancing liver mitochondrial fatty acid oxidation capacity in obese mice improves insulin sensitivity independently of hepatic steatosis. J Hepatol. 2012;56(3):632-639.

40. Sunny NE, Bril F, Cusi K. Mitochondrial adaptation in nonalcoholic fatty liver disease: novel mechanisms and treatment strategies. Trends Endocrinol Metab. 2017;28(4):250-260.

41. McCurdy CE, et al. Maternal high-fat diet triggers lipotoxicity in the fetal livers of nonhuman primates. JClin Invest. 2009;119(2):323-335.

42. Thorn SR, et al. Early life exposure to maternal insulin resistance has persistent effects on hepatic NAFLD in juvenile nonhuman primates. Diabetes. 2014;63(8):2702-2713.

43. Grant WF, et al. Maternal high fat diet is associated with decreased plasma $\mathrm{n}-3$ fatty acids and fetal hepatic apoptosis in nonhuman primates. PLoS One. 2011;6(2):e17261.

44. Pathil A, Liebisch G, Okun JG, Chamulitrat W, Schmitz G, Stremmel W. Ursodeoxycholyl lysophosphatidylethanolamide modifies aberrant lipid profiles in NAFLD. Eur JClin Invest. 2015;45(9):925-931.

45. Feldstein AE, Canbay A, Guicciardi ME, Higuchi $\mathrm{H}$, Bronk SF, Gores GJ. Diet associated hepatic steatosis sensitizes to Fas mediated liver injury in mice. J Hepatol. 2003;39(6):978-983.

46. Malhi H, Barreyro FJ, Isomoto H, Bronk SF, Gores GJ. Free fatty acids sensitise hepatocytes to TRAIL mediated cytotoxicity. Gut.
2007;56(8):1124-1131.

47. Friedman JE, et al. Pyrroloquinoline quinone prevents developmental programming of microbial dysbiosis and macrophage polarization to attenuate liver fibrosis in offspring of obese mice. Hepatol Commun. 2018;2(3):313-328.

48. Gregorio BM, Souza-Mello V, Carvalho JJ, Mandarim-de-Lacerda CA, Aguila MB. Maternal high-fat intake predisposes nonalcoholic fatty liver disease in C57BL/6 offspring. Am JObstet Gynecol. 2010;203(5):495.e1-495.e8.

49. Alfaradhi MZ, Fernandez-Twinn DS, Martin-Gronert MS, Musial B, Fowden A, Ozanne SE. Oxidative stress and altered lipid homeostasis in the programming of offspring fatty liver by maternal obesity. Am J Physiol Regul Integr Comp Physiol. 2014;307(1):R26-R34.

50. Wankhade UD, et al. Enhanced offspring predisposition to steatohepatitis with maternal high-fat diet is associated with epigenetic and microbiome alterations. PLoS One. 2017;12(4):e0175675.

51. Bruce KD, et al. Maternal high-fat feeding primes steatohepatitis in adult mice offspring, involving mitochondrial dysfunction and altered lipogenesis gene expression. Hepatology. 2009;50(6):1796-1808.

52. Pruis MG, et al. Maternal western diet primes non-alcoholic fatty liver disease in adult mouse offspring. Acta Physiol (Oxf). 2014;210(1):215-227.

53. Mouralidarane A, et al. Maternal obesity programs offspring nonalcoholic fatty liver disease by innate immune dysfunction in mice. Hepatology. 2013;58(1):128-138.

54. Burgueño AL, Cabrerizo R, Gonzales Mansilla N, Sookoian S, Pirola CJ. Maternal high-fat intake during pregnancy programs metabolic-syndrome-related phenotypes through liver mitochondrial DNA copy number and transcriptional activity of liver PPARGC1A. J Nutr Biochem. 2013;24(1):6-13.

55. Hellgren LI, Jensen RI, Waterstradt MS, Quistorff B, Lauritzen L. Acute and perinatal programming effects of a fat-rich diet on rat muscle mitochondrial function and hepatic lipid accumulation. Acta Obstet Gynecol Scand. 2014;93(11):1170-1180.

56. Yamada M, Wolfe D, Han G, French SW, Ross MG, Desai M. Early onset of fatty liver in growth-restricted rat fetuses and newborns. Congenit Anom (Kyoto). 2011;51(4):167-173.

57. Su YM, Lv GR, Xie JX, Wang ZH, Lin HT. Maternal hypoxia increases the susceptibility of adult rat male offspring to high-fat diet-induced nonalcoholic fatty liver disease. Endocrinology. 2013;154(11):4377-4387.

58. Wolfe D, Gong M, Han G, Magee TR, Ross MG, Desai M. Nutrient sensor-mediated programmed nonalcoholic fatty liver disease in low birthweight offspring. Am JObstet Gynecol. 2012;207(4):308.e1-308.e6.

59. Cao L, et al. Hepatic insulin signaling changes: possible mechanism in prenatal hypoxia-increased susceptibility of fatty liver in adulthood. Endocrinology. 2012;153(10):4955-4965.

60. Magee TR, Han G, Cherian B, Khorram O, Ross MG, Desai M. Down-regulation of transcription factor peroxisome proliferator-activated receptor in programmed hepatic lipid dysregulation and inflammation in intrauterine growth-restricted offspring. $\mathrm{Am} J$ 
Obstet Gynecol. 2008;199(3):271.e1-271.e5.

61. Seki Y, et al. In utero exposure to a high-fat diet programs hepatic hypermethylation and gene dysregulation and development of metabolic syndrome in male mice. Endocrinology. 2017;158(9):2860-2872.

62. Suter MA, et al. In utero exposure to a maternal high-fat diet alters the epigenetic histone code in a murine model. Am JObstet Gynecol. 2014;210(5):463.e1-463.e11.

63. Wang S, Song J, Yang Y, Zhang Y, Wang H, Ma J. HIF3A DNA methylation is associated with childhood obesity and ALT. PLoS One. 2015;10(12):e0145944.

64. Hotta K, et al. Identification of the genomic region under epigenetic regulation during non-alcoholic fatty liver disease progression. Hepatol Res. 2018;48(3):E320-E334.

65. Pirola CJ, et al. Epigenetic modification of liver mitochondrial DNA is associated with histological severity of nonalcoholic fatty liver disease. Gut. 2013;62(9):1356-1363.

66. Pirola CJ, et al. Epigenetic modifications in the biology of nonalcoholic fatty liver disease: the role of DNA hydroxymethylation and TET proteins. Medicine (Baltimore). 2015;94(36):e1480.

67. Sookoian S, et al. Epigenetic regulation of insulin resistance in nonalcoholic fatty liver disease: impact of liver methylation of the peroxisome proliferator-activated receptor $\gamma$ coactivator $1 \alpha$ promoter. Hepatology. 2010;52(6):1992-2000.

68. Suter MA, et al. A maternal high-fat diet modulates fetal SIRT1 histone and protein deacetylase activity in nonhuman primates. FASEB J. 2012;26(12):5106-5114.

69. Tobita T, et al. SIRT1 disruption in human fetal hepatocytes leads to increased accumulation of glucose and lipids. PLoS One. 2016;11(2):e0149344.

70. Romeo S, et al. The $148 \mathrm{M}$ allele of the PNPLA3 gene is associated with indices of liver damage early in life. J Hepatol. 2010;53(2):335-338.

71. Lotowska JM, Sobaniec-Lotowska ME, Bockowska SB, Lebensztejn DM. Pediatric non-alcoholic steatohepatitis: the first report on the ultrastructure of hepatocyte mitochondria. World J Gastroenterol. 2014;20(15):4335-4340.

72. Chedid A, et al. Significance of megamitochondria in alcoholic liver disease. Gastroenterology. 1986;90(6):1858-1864.

73. Wieczorek A, Stępień PM, Zarębska-Michaluk D, Kryczka W, Pabjan P, Król T. Megamitochondria formation in hepatocytes of patient with chronic hepatitis C - a case report. Clin Exp Hepatol. 2017;3(3):169-175.

74. Sanyal AJ, et al. Nonalcoholic steatohepatitis: association of insulin resistance and mitochondrial abnormalities. Gastroenterology. 2001;120(5):1183-1192.

75. Nakata R, et al. In vivo redox metabolic imaging of mitochondria assesses disease progression in non-alcoholic steatohepatitis. Sci Rep. 2017;7(1):17170.

76. Sobaniec-Lotowska ME, Lebensztejn DM. Ultrastructure of hepatocyte mitochondria in nonalcoholic steatohepatitis in pediatric patients: usefulness of electron microscopy in the diagnosis of the disease. Am J Gastroenterol.
2003;98(7):1664-1665.

77. Wree A, Kahraman A, Gerken G, Canbay A. Obesity affects the liver - the link between adipocytes and hepatocytes. Digestion. 2011;83(1-2):124-133.

78. Browning JD, Horton JD. Molecular mediators of hepatic steatosis and liver injury. J Clin Invest. 2004;114(2):147-152.

79. Caldwell SH, Chang CY, Nakamoto RK, Krugner-Higby L. Mitochondria in nonalcoholic fatty liver disease. Clin Liver Dis. 2004;8(3):595-617.

80. Pérez-Carreras M, et al. Defective hepatic mitochondrial respiratory chain in patients with nonalcoholic steatohepatitis. Hepatology. 2003;38(4):999-1007.

81. Begriche K, Massart J, Robin MA, Bonnet F, Fromenty B. Mitochondrial adaptations and dysfunctions in nonalcoholic fatty liver disease. Hepatology. 2013;58(4):1497-1507.

82. Satapati S, et al. Mitochondrial metabolism mediates oxidative stress and inflammation in fatty liver. JClin Invest. 2015;125(12):4447-4462.

83. Seifert EL, Estey C, Xuan JY, Harper ME. Electron transport chain-dependent and -independent mechanisms of mitochondrial $\mathrm{H} 2 \mathrm{O} 2$ emission during long-chain fatty acid oxidation. J Biol Chem. 2010;285(8):5748-5758.

84. Hodson L, et al. Greater dietary fat oxidation in obese compared with lean men: an adaptive mechanism to prevent liver fat accumulation? Am JPhysi ol Endocrinol Metab. 2010;299(4):E584-E592.

85. Miele L, et al. Hepatic mitochondrial beta-oxidation in patients with nonalcoholic steatohepatitis assessed by $13 \mathrm{C}$-octanoate breath test. Am J Gastroenterol. 2003;98(10):2335-2336.

86. Gao Q, et al. PPAR $\alpha$-deficient ob/ob obese mice become more obese and manifest severe hepatic steatosis due to decreased fatty acid oxidation. Am J Pathol. 2015;185(5):1396-1408.

87. Memon RA, et al. Up-regulation of peroxisome proliferator-activated receptors (PPAR- $\alpha$ ) and PPAR- $\gamma$ messenger ribonucleic acid expression in the liver in murine obesity: troglitazone induces expression of PPAR- $\gamma$-responsive adipose tissue-specific genes in the liver of obese diabetic mice. Endocrinology. 2000;141(11):4021-4031.

88. Lee $\mathrm{Y}$, et al. PPAR alpha is necessary for the lipopenic action of hyperleptinemia on white adipose and liver tissue. Proc Natl Acad Sci U S A. 2002;99(18):11848-11853.

89. Kim HJ, et al. Liver-specific deletion of ROR $\alpha$ aggravates diet-induced nonalcoholic steatohepatitis by inducing mitochondrial dysfunction. $S c i$ Rep. 2017;7(1):16041.

90. Takamura $\mathrm{T}$, et al. Obesity upregulates genes involved in oxidative phosphorylation in livers of diabetic patients. Obesity (Silver Spring). 2008;16(12):2601-2609.

91. Stefanovic-Racic M, Perdomo G, Mantell BS, Sipula IJ, Brown NF, O’Doherty RM. A moderate increase in carnitine palmitoyltransferase 1a activity is sufficient to substantially reduce hepatic triglyceride levels. Am J Physiol Endocrinol Metab. 2008;294(5):E969-E977.

92. Szendroedi J, et al. Abnormal hepatic energy homeostasis in type 2 diabetes. Hepatology. 2009;50(4):1079-1086.

93. Morris EM, et al. Aerobic capacity and hepatic mitochondrial lipid oxidation alters suscepti- bility for chronic high-fat diet-induced hepatic steatosis. Am J Physiol Endocrinol Metab. 2016;311(4):E749-E760.

94. Sunny NE, Parks EJ, Browning JD, Burgess SC. Excessive hepatic mitochondrial TCA cycle and gluconeogenesis in humans with nonalcoholic fatty liver disease. Cell Metab. 2011;14(6):804-810.

95. Satapati S, et al. Elevated TCA cycle function in the pathology of diet-induced hepatic insulin resistance and fatty liver. J Lipid Res. 2012;53(6):1080-1092.

96. Franko A, et al. Liver adapts mitochondrial function to insulin resistant and diabetic states in mice. J Hepatol. 2014;60(4):816-823.

97. Portincasa P, Grattagliano I, Lauterburg BH, Palmieri VO, Palasciano G, Stellaard F. Liver breath tests non-invasively predict higher stages of non-alcoholic steatohepatitis. Clin Sci. 2006;111(2):135-143.

98. Ruiz JR, Lasa A, Simon E, Larrarte E, Labayen I. Lower plasma NAMPT/visfatin levels are associated with impaired hepatic mitochondrial function in non-diabetic obese women: a potential link between obesity and non-alcoholic fatty liver disease. Nutr Metab Cardiovasc Dis. 2012;22(2):e1-e2.

99. Mion F, Rousseau M, Brazier JL, Minaire Y. Human hepatic macrovesicular steatosis: a noninvasive study of mitochondrial ketoisocaproic acid decarboxylation. Metab Clin Exp. 1995;44(6):699-700.

100.Newgard CB. Interplay between lipids and branched-chain amino acids in development of insulin resistance. Cell Metab. 2012;15(5):606-614.

101.Lake AD, et al. Branched chain amino acid metabolism profiles in progressive human nonalcoholic fatty liver disease. Amino Acids. 2015;47(3):603-615.

102. Cheng $\mathrm{S}$, et al. Adipose tissue dysfunction and altered systemic amino acid metabolism are associated with non-alcoholic fatty liver disease. PLoS One. 2015;10(10):e0138889.

103. Goffredo M, et al. A branched-chain amino acid-related metabolic signature characterizes obese adolescents with non-alcoholic fatty liver disease. Nutrients. 2017;9(7):E642.

104.Yang W, et al. Integrative transcriptomic analysis of NAFLD animal model reveals dysregulated genes and pathways in metabolism. Gene. 2016;595(1):99-108.

105. Pogribny IP, et al. Epigenetically mediated inhibition of S-adenosylhomocysteine hydrolase and the associated dysregulation of 1-carbon metabolism in nonalcoholic steatohepatitis and hepatocellular carcinoma. FASEB J. 2018;32(3):1591-1601.

106. Murphy SK, et al. Relationship between methylome and transcriptome in patients with nonalcoholic fatty liver disease. Gastroenterology. 2013;145(5):1076-1087.

107. Marcolin E, Forgiarini LF, Tieppo J, Dias AS, Freitas LA, Marroni NP. Methionine- and choline-deficient diet induces hepatic changes characteristic of non-alcoholic steatohepatitis. Arq Gastroenterol. 2011;48(1):72-79.

108. Hensley K, et al. Dietary choline restriction causes complex I dysfunction and increased $\mathrm{H}(2) \mathrm{O}(2)$ generation in liver mitochondria. Carcinogenesis. 2000;21(5):983-989. 
109.Petrosillo G, et al. Mitochondrial dysfunction in rat with nonalcoholic fatty liver involvement of complex I, reactive oxygen species and cardiolipin. Biochim Biophys Acta. 2007;1767(10):1260-1267.

110.Teodoro JS, Rolo AP, Duarte FV, Simões AM, Palmeira CM. Differential alterations in mitochondrial function induced by a choline-deficient diet: understanding fatty liver disease progression. Mitochondrion. 2008;8(5-6):367-376.

111. Caballero F, et al. Specific contribution of methionine and choline in nutritional nonalcoholic steatohepatitis: impact on mitochondrial S-adenosyl-L-methionine and glutathione. J Biol Chem. 2010;285(24):18528-18536.

112. Rinella ME, Elias MS, Smolak RR, Fu T, Borensztajn J, Green RM. Mechanisms of hepatic steatosis in mice fed a lipogenic methionine choline-deficient diet. JLipid Res. 2008;49(5):1068-1076.

113. Paradies G, Paradies V, Ruggiero FM, Petrosillo G. Oxidative stress, cardiolipin and mitochondrial dysfunction in nonalcoholic fatty liver disease. World J Gastroenterol. 2014;20(39):14205-14218.

114. Mato JM, Martinez-Chantar ML, Lu SC. Methionine metabolism and liver disease. Annu Rev Nutr. 2008;28:273-293.

115. Videla LA, et al. Oxidative stress-related parameters in the liver of non-alcoholic fatty liver disease patients. Clin Sci (Lond). 2004;106(3):261-268.

116. Qanungo S, Mukherjea M. Ontogenic profile of some antioxidants and lipid peroxidation in human placental and fetal tissues. Mol Cell Biochem. 2000;215(1-2):11-19.

117. Du J, et al. Pro-inflammatory CXCR3 impairs mitochondrial function in experimental non-alcoholic steatohepatitis. Theranostics. 2017;7(17):4192-4203.

118. Mantena SK, et al. High fat diet induces dysregulation of hepatic oxygen gradients and mitochondrial function in vivo. Biochem $J$. 2009;417(1):183-193.

119. García-Ruiz I, et al. Uric acid and anti-TNF antibody improve mitochondrial dysfunction in ob/ ob mice. Hepatology. 2006;44(3):581-591.

120. Marí M, et al. Mitochondrial free cholesterol loading sensitizes to TNF- and Fas-mediated steatohepatitis. Cell Metab. 2006;4(3):185-198.

121. Frias AE, et al. Maternal high-fat diet disturbs uteroplacental hemodynamics and increases the frequency of stillbirth in a nonhuman primate model of excess nutrition. Endocrinology. 2011;152(6):2456-2464.

122. Bellanti F, et al. Synergistic interaction of fatty acids and oxysterols impairs mitochondrial function and limits liver adaptation during nafld progression. Redox Biol. 2018;15:86-96.

123. Cazanave SC, et al. JNK1-dependent PUMA expression contributes to hepatocyte lipoapoptosis. J Biol Chem. 2009;284(39):26591-26602.

124. Bruey JM, et al. Bcl-2 and Bcl-XL regulate proinflammatory caspase- 1 activation by interaction with NALP1. Cell. 2007;129(1):45-56.

125. Malhi H, Bronk SF, Werneburg NW, Gores GJ. Free fatty acids induce JNK-dependent hepatocyte lipoapoptosis. J Biol Chem. 2006;281(17):12093-12101.

126. Barreyro FJ, Kobayashi S, Bronk SF, Werneburg NW, Malhi H, Gores GJ. Transcriptional regulation of Bim by FoxO3A mediates hepatocyte lipoapop- tosis. J Biol Chem. 2007;282(37):27141-27154.

127. Nolan CJ, Larter CZ. Lipotoxicity: why do saturated fatty acids cause and monounsaturates protect against it? J Gastroenterol Hepatol. 2009;24(5):703-706.

128. Li ZZ, Berk M, McIntyre TM, Feldstein AE. Hepatic lipid partitioning and liver damage in nonalcoholic fatty liver disease: role of stearoyl-CoA desaturase. J Biol Chem. 2009;284(9):5637-5644.

129. Bechmann LP, et al. Apoptosis is associated with CD36/fatty acid translocase upregulation in non-alcoholic steatohepatitis. Liver Int. 2010;30(6):850-859.

130. Pagadala M, Kasumov T, McCullough AJ, Zein NN, Kirwan JP. Role of ceramides in nonalcoholic fatty liver disease. Trends Endocrinol Metab. 2012;23(8):365-371.

131. Holland WL, Summers SA. Sphingolipids, insulin resistance, and metabolic disease: new insights from in vivo manipulation of sphingolipid metabolism. Endocr Rev. 2008;29(4):381-402.

132. Raichur S, et al. CerS2 haploinsufficiency inhibits $\beta$-oxidation and confers susceptibility to diet-induced steatohepatitis and insulin resistance. Cell Metab. 2014;20(4):687-695.

133. Alkhouri N, et al. Development and validation of a new histological score for pediatric non-alcoholic fatty liver disease. J Hepatol. 2012;57(6):1312-1318.

134. Giorgio V, Prono F, Graziano F, Nobili V. Pediatric non alcoholic fatty liver disease: old and new concepts on development, progression, metabolic insight and potential treatment targets. $B M C$ Pediatr. 2013;13:40.

135. Uppal V, Mansoor S, Furuya KN. Pediatric non-alcoholic fatty liver disease. Curr Gastroenterol Rep. 2016;18(5):24.

136. Perng W, et al. Associations of cord blood metabolites with perinatal characteristics, newborn anthropometry, and cord blood hormones in project viva. Metab Clin Exp. 2017;76:11-22.

137. Simpson J, et al. Cord blood adipokines and lipids and adolescent nonalcoholic fatty liver disease. JClin Endocrinol Metab. 2016;101(12):4661-4668.

138. Jin R, et al. Amino acid metabolism is altered in adolescents with nonalcoholic fatty liver disease-an untargeted, high resolution metabolomics study. J Pediatr. 2016;172:14-19.e5.

139. Pastore A, et al. Plasma levels of homocysteine and cysteine increased in pediatric NAFLD and strongly correlated with severity of liver damage. Int JMol Sci. 2014;15(11):21202-21214.

140.Wahl S, et al. Childhood obesity is associated with changes in the serum metabolite profile. Obes Facts. 2012;5(5):660-670.

141. McCormack SE, et al. Circulating branched-chain amino acid concentrations are associated with obesity and future insulin resistance in children and adolescents. Pediatr Obes. 2013;8(1):52-61.

142.Baker P II, Ayres L, Gaughan S, Weisfeld-Adams J. Hereditary fructose intolerance. In: Adam MP, et al., eds. GeneReviews. Seattle, Washington, USA: University of Washington; 2015.

143. Alwahsh SM, Gebhardt R. Dietary fructose as a risk factor for non-alcoholic fatty liver disease (NAFLD). Arch Toxicol. 2017;91(4):1545-1563.

144. Cortez-Pinto H, Chatham J, Chacko VP, Arnold C, Rashid A, Diehl AM. Alterations in liver ATP homeostasis in human nonalcoholic steatohepatitis: a pilot study. JAMA. 1999;282(17):1659-1664.

145. Lim JS, Mietus-Snyder M, Valente A, Schwarz JM, Lustig RH. The role of fructose in the pathogenesis of NAFLD and the metabolic syndrome. Nat Rev Gastroenterol Hepatol. 2010;7(5):251-264.

146.Yamazaki M, et al. Fructose consumption induces hypomethylation of hepatic mitochondrial DNA in rats. Life Sci. 2016;149:146-152.

147. Drew L. Drug development: sprint finish. Nature. 2017;551(7681):S86-S89.

148. Catalano PM, Shankar K. Obesity and pregnancy: mechanisms of short term and long term adverse consequences for mother and child. BMJ. 2017;356:j1.

149. Hernandez TL, et al. Women with gestational diabetes mellitus randomized to a higher-complex carbohydrate/low-fat diet manifest lower adipose tissue insulin resistance, inflammation, glucose, and free fatty acids: a pilot study. Diabetes Care. 2016;39(1):39-42.

150. Nobili V, et al. A protective effect of breastfeeding on the progression of non-alcoholic fatty liver disease. Arch Dis Child. 2009;94(10):801-805.

151. Félix DR, Costenaro F, Gottschall CB, Coral GP. Non-alcoholic fatty liver disease (Nafld) in obese children-effect of refined carbohydrates in diet. BMC Pediatr. 2016;16(1):187.

152. Okada LSDRR, et al. Omega-3 PUFA modulate lipogenesis, ER stress, and mitochondrial dysfunction markers in NASH - proteomic and lipidomic insight [published online ahead of print September 7, 2017]. Clin Nutr. https://doi.org/10.1016/j.clnu.2017.08.031.

153. Mitchell AE, Jones AD, Mercer RS, Rucker RB. Characterization of pyrroloquinoline quinone amino acid derivatives by electrospray ionization mass spectrometry and detection in human milk. Anal Biochem. 1999;269(2):317-325.

154. Sheldon RD, et al. Gestational exercise protects adult male offspring from high-fat diet-induced hepatic steatosis. J Hepatol. 2016;64(1):171-178.

155. van der Windt DJ, Sud V, Zhang H, Tsung A, Huang $H$. The effects of physical exercise on fatty liver disease. Gene Expr. 2018;18(2):89-101.

156.Akcam M, Boyaci A, Pirgon O, Kaya S, Uysal S, Dundar BN. Therapeutic effect of metformin and vitamin $E$ versus prescriptive diet in obese adolescents with fatty liver. Int J Vitam Nutr Res. 2011;81(6):398-406.

157. Lavine JE, et al. Effect of vitamin $\mathrm{E}$ or metformin for treatment of nonalcoholic fatty liver disease in children and adolescents: the TONIC randomized controlled trial. JAMA. 2011;305(16):1659-1668.

158. Nobili V, et al. Metformin use in children with nonalcoholic fatty liver disease: an open-label, 24-month, observational pilot study. Clin Ther. 2008;30(6):1168-1176.

159. Lindsay RS, Loeken MR. Metformin use in pregnancy: promises and uncertainties. Diabetologia. 2017;60(9):1612-1619.

160.Larrieta-Carrasco E, et al. Genetic variants in COL13A1, ADIPOQ and SAMM50, in addition to the PNPLA3 gene, confer susceptibility to elevated transaminase levels in an admixed Mexican population. Exp Mol Pathol. 2018;104(1):50-58.

161. Coletta DK, et al. Pioglitazone stimulates AMP-activated protein kinase signalling and increases the 
expression of genes involved in adiponectin signalling, mitochondrial function and fat oxidation in human skeletal muscle in vivo: a randomised trial. Diabetologia. 2009;52(4):723-732.

162.Lin YC, Chang PF, Chang MH, Ni YH. Genetic determinants of hepatic steatosis and serum cytokeratin-18 fragment levels in Taiwanese children. Liver Int. 2018;38(7):1300-1307.

163. Lee JW, et al. Mitochondrial dysfunction: glucokinase downregulation lowers interaction of glucokinase with mitochondria, resulting in apoptosis of pancreatic beta-cells. Cell Signal. 2009;21(1):69-78.

164. Li H, et al. Long term liver specific glucokinase gene defect induced diabetic cardiomyopathy by up regulating NADPH oxidase and down regulating insulin receptor and p-AMPK. Cardiovasc Diabetol. 2014;13:24.

165. Wang S, et al. Interaction between obesity and the Hypoxia Inducible Factor 3 Alpha Subunit rs3826795 polymorphism in relation with plasma alanine aminotransferase. BMC Med Genet 2017;18(1):80.

166. Liu Y, et al. HIF- $1 \alpha$ and HIF- $2 \alpha$ are critically involved in hypoxia-induced lipid accumulation in hepatocytes through reducing PGC-1 $\alpha$-mediated fatty acid $\beta$-oxidation. Toxicol Lett. 2014;226(2):117-123.

167. Hsu CC, et al. Mitochondrial dysfunction represses HIF- $1 \alpha$ protein synthesis through AMPK activation in human hepatoma HepG2 cells. Biochim Biophys Acta . 2013;1830(10):4743-4751.

168. Anavi S, Hahn-Obercyger M, Madar Z, Tirosh O. Mechanism for HIF-1 activation by cholesterol under normoxia: a redox signaling pathway for liver damage. Free Radic Biol Med. 2014;71:61-69.

169. Goda N, Kanai M. Hypoxia-inducible factors and their roles in energy metabolism. Int J Hematol. 2012;95(5):457-463.

170.Chang PF, Lin YC, Liu K, Yeh SJ, Ni YH. Heme oxygenase-1 gene promoter polymorphism and the risk of pediatric nonalcoholic fatty liver disease. Int JObes (Lond). 2015;39(8):1236-1240.

171. Cheng $\mathrm{Z}$, et al. Foxo1 integrates insulin signaling with mitochondrial function in the liver. Nat Med. 2009;15(11):1307-1311.

172. Chen Y, et al. Synergistic effects of cilostazol and probucol on ER stress-induced hepatic steatosis via heme oxygenase-1-dependent activation of mitochondrial biogenesis. Oxid Med Cell Longev. 2016;2016:3949813

173. Lee SJ, Zhang J, Choi AM, Kim HP. Mitochondrial dysfunction induces formation of lipid droplets as a generalized response to stress. Oxid Med Cell
Longev. 2013;2013:327167.

174. Liu X, et al. Sirt1 mediates the effect of the heme oxygenase inducer, cobalt protoporphyrin, on ameliorating liver metabolic damage caused by a high-fat diet. J Hepatol. 2015;63(3):713-721.

175. Valenti L, et al. LPIN1 rs13412852 polymorphism in pediatric nonalcoholic fatty liver disease. J Pediatr Gastroenterol Nutr. 2012;54(5):588-593.

176. Assaily W, et al. ROS-mediated p53 induction of Lpin1 regulates fatty acid oxidation in response to nutritional stress. Mol Cell. 2011;44(3):491-501.

177. Viitasalo A, Eloranta AM, Atalay M, Romeo S, Pihlajamäki J, Lakka TA. Association of MBOAT7 gene variant with plasma ALT levels in children: the PANIC study. Pediatr Res. 2016;80(5):651-655.

178. Hirata Y, Yamamori N, Kono N, Lee HC, Inoue T, Arai H. Identification of small subunit of serine palmitoyltransferase a as a lysophosphatidylinositol acyltransferase 1-interacting protein. Genes Cells. 2013;18(5):397-409.

179. El-Koofy NM, El-Karaksy HM, Mandour IM, Anwar GM, El-Raziky MS, El-Hennawy AM. Genetic polymorphisms in non-alcoholic fatty liver disease in obese Egyptian children. Saudi J Gastroenterol. 2011;17(4):265-270.

180. Krautbauer S, et al. Manganese superoxide dismutase is reduced in the liver of male but not female humans and rodents with non-alcoholic fatty liver disease. Exp Mol Pathol. 2013;95(3):330-335.

181. Perlemuter G, et al. Increase in liver antioxidant enzyme activities in non-alcoholic fatty liver disease. Liver Int. 2005;25(5):946-953.

182. Candas D, Li JJ. MnSOD in oxidative stress response-potential regulation via mitochondrial protein influx. Antioxid Redox Signal. 2014;20(10):1599-1617.

183. Gong Z, et al. Central effects of humanin on hepatic triglyceride secretion. Am J Physiol Endocrinol Metab. 2015;309(3):E283-E292.

184. Tang J, et al. Severe riboflavin deficiency induces alterations in the hepatic proteome of starter Pekin ducks. Br J Nutr. 2017;118(9):641-650.

185. Miotto PM, et al. $\alpha$-Linolenic acid supplementation and exercise training reveal independent and additive responses on hepatic lipid accumulation in obese rats. Am J Physiol Endocrinol Metab. 2017;312(6):E461-E470.

186.Ko KS, et al. Liver-specific deletion of prohibitin 1 results in spontaneous liver injury, fibrosis, and hepatocellular carcinoma in mice. Hepatology. 2010;52(6):2096-2108.

187. Ande SR, Nguyen KH, Grégoire Nyomba BL, Mishra S. Prohibitin-induced, obesity-associated insulin resistance and accompanying low-grade inflammation causes NASH and HCC. Sci Rep. 2016;6:23608.

188. Mishra S, Murphy LC, Murphy LJ. The Prohibitins: emerging roles in diverse functions. J Cell Mol Med. 2006;10(2):353-363.

189. Lin YC, Chang PF, Chang MH, Ni YH. A common variant in the peroxisome proliferator-activated receptor- $\gamma$ coactivator- $1 \alpha$ gene is associated with nonalcoholic fatty liver disease in obese children. Am J Clin Nutr. 2013;97(2):326-331.

190. Chung GE, et al. Genetic polymorphisms of PNPLA3 and SAMM50 are associated with nonalcoholic fatty liver disease in a Korean population. Gut Liver. 2018;12(3):316-323.

191. Ott C, et al. Sam50 functions in mitochondrial intermembrane space bridging and biogenesis of respiratory complexes. Mol Cell Biol. 2012;32(6):1173-1188.

192. Ott C, Dorsch E, Fraunholz M, Straub S, Kozjak-Pavlovic V. Detailed analysis of the human mitochondrial contact site complex indicate a hierarchy of subunits. PLoS One. 2015;10(3):e0120213.

193. Goffredo M, et al. Role of TM6SF2 rs58542926 in the pathogenesis of nonalcoholic pediatric fatty liver disease: a multiethnic study. Hepatology. 2016;63(1):117-125

194. Grandone A, et al. TM6SF2 Glu167Lys polymor phism is associated with low levels of LDL-cholesterol and increased liver injury in obese children. Pediatr Obes. 2016;11(2):115-119.

195. Mahdessian $\mathrm{H}$, et al. TM6SF2 is a regulator of liver fat metabolism influencing triglyceride secretion and hepatic lipid droplet content. Proc Natl Acad Sci U S A. 2014;111(24):8913-8918.

196. Smagris E, Gilyard S, BasuRay S, Cohen JC, Hobbs HH. Inactivation of Tm6sf2, a gene defective in fatty liver disease, impairs lipidation but not secretion of very low density lipoproteins. J Biol Chem. 2016;291(20):10659-10676.

197. Fares R, et al. The UCP2-866 G>A promoter region polymorphism is associated with nonalcoholic steatohepatitis. Liver Int. 2015;35(5):1574-1580.

198. Baffy G. Uncoupling protein-2 and non-alcoholic fatty liver disease. Front Biosci. 2005;10:2082-2096

199.Lin L, Guan X, Wang L, Tang Y. [Influence of genipin and vitamin E on UCP2 and other correlation factors in non-alcoholic fatty liver disease]. Sheng Wu Yi Xue Gong Cheng Xue Za Zhi. 2010;27(6):1327-1331.

200.Kohjima M, et al. Re-evaluation of fatty acid metabolism-related gene expression in nonalcoholic fatty liver disease. Int J Mol Med. 2007;20(3):351-358. 